\title{
The Challenge of Treating Children with Hepatitis C Virus Infection
}

\author{
Giuseppe Indolfi ${ }^{1}$, Claire Thorne ${ }^{2}$, Manal H. El Sayed ${ }^{3}$, \\ Carlo Giaquinto ${ }^{4,5}$, Regino P. Gonzalez-Peralta ${ }^{6}$
}

${ }^{1}$ Paediatric and Liver Unit, Meyer Children's University Hospital of Florence, Italy; ${ }^{2} \mathrm{UCL}$ Institute of Child Health, University College London, UK; ${ }^{3}$ Department of Pediatrics, Faculty of Medicine, Ain Shams University, Cairo, Egypt; ${ }^{4}$ Department of Women and Child Health, University of Padua, Italy; ${ }^{5}$ PENTA Foundation, Padua, Italy; ${ }^{6}$ Division of Gastroenterology, Hepatology and Nutrition, Department of Paediatrics, University of Florida College of Medicine, Gainesville, FL, US

Corresponding author

Dr Giuseppe Indolfi. Paediatric and Liver Unit, Meyer Children's University Hospital of Florence. Viale Gaetano Pieraccini 34, I-50139 Firenze, Italy. Fax +39055 5662400; tel +39055 5662480. E-mail: g.indolfi@meyer.it

Keyword

Hepatitis C virus; chronic viral hepatitis; direct acting antiviral therapy, pediatric hepatology; pediatric liver disease 


\section{Competing interests and funding}

Regino P Gonzalez-Peralta receives research grants from Gilead, Abbvie, Target NASH, Braintree and Sucampo and he serves on Safety Monitoring Boards of Shire and GenetechRoche and Scientific Advisory Board of Retrophin, Albireo and Horizon. Claire Thorne has received funding from Abbvie. Giuseppe Indolfi has served on Scientific Advisory Board of Alexion.

\section{Contributing authorship statement}

All the authors contributed equally to the paper.

Acknowledgement: We thank Joel M. Andres, MD for critically reviewing this manuscript. 


\section{Learning points}

1. More than eleven million children are anti-HCV antibody positive worldwide and half of them are viremic needing treatment.

2. Pegylated interferon in combination with ribavirin is the only currently approved therapy for children with HCV infection. This therapeutic regime is suboptimally effective, requires subcutaneous injections, and is commonly associated with treatment-related adverse events.

3. Newly developed direct acting antivirals (DAAs) are highly effective and safe drugs to treat HCV but are currently only approved for use in adults. Preliminary data from ongoing pediatric trials show that DAAs are similarly effective and safe in children and adolescents.

4. Cure of HCV infection is possible in almost all patients treated with interferon-free, oral DAA regimens. HCV- infected children need to be targeted for DAA treatment as an integral component of the public health approach to the global eradication of $\mathrm{HCV}$ infection.

5. Serious interplay between pediatric researchers, regulators and pharmaceutical industry leaders is essential to hasten the process of DAA approval for children with HCV infection. 


\begin{abstract}
The development of oral hepatitis $\mathrm{C}$ virus direct acting antivirals has revolutionized the therapeutic field. Nowadays, multiple safe and highly effective antiviral regimens are commercially available to treat adults with hepatitis $\mathrm{C}$ infection. These new regimens for the first time, genuinely raise the prospects of eradicating hepatitis $\mathrm{C}$ virus. However, many challenges remain: from identifying infected individuals to optimizing treatment and ensuring global access to antiviral therapy to all population groups, including children. The only drugs currently approved for children with chronic hepatitis $\mathrm{C}$ virus infection are pegylated interferon and ribavirin. There are four registered ongoing paediatric trials assessing safety and efficacy of direct acting antivirals but their current completion timelines are years away. Herein, we summarize the state of the art of direct acting antivirals development for adult and children and highlight the crucial importance of overcoming barriers to treating children with hepatitis $\mathrm{C}$ virus.
\end{abstract}




\section{Introduction}

The discovery of hepatitis $\mathrm{C}$ virus (HCV) 25 years ago was a major scientific achievement when this elusive virus was isolated via a new expression-cloning technique (1). Subsequent molecular studies revealed HCV to be an RNA virus that replicates without a DNA intermediate (2). The characterization of HCV non-structural proteins (NS3-5), responsible for viral replication allowed for development of direct acting antiviral (DAA) targeting of NS3/4A protease, NS5A, and both nucleoside and non-nucleoside NS5B polymerase (3). Since the introduction of the first generation DAAs (i.e., protease inhibitors) in 2011, HCV therapy has become one of the great success stories of basic, clinical and translational viral research. Hence, our focus is to outline the development of DAAs; and, to review HCV oral, non-interferon therapy for adults, older adolescents and children. Also, we will highlight pediatric barriers that must be overcome to allow children with chronic hepatitis to participate in this rapidly evolving post-interferon era which has revolutionized HCV therapy.

\section{Prevalence of Hepatitis C Virus}

Chronic HCV is a major cause of liver cirrhosis and hepatocellular carcinoma (HCC) worldwide. Throughout this world, 130 million to 170 million people are chronically infected with HCV. In the United States (US) prevalence is estimated to be 3.2 million individuals (4). Hepatitis $\mathrm{C}$ virus infection recently eclipsed human immunodeficiency virus infection as a cause of death in the US; the number of deaths because of HCV is not projected to peak until 2030 (5). Globally, 11 million of these infected individuals are $<15$ years of age; greater than $50 \%$ of all the children are from China, India, Russia, Nigeria, Pakistan, and Egypt (6). Hepatitis C virus prevalence in the US and Europe ranges from 0.05 to $0.36 \%$, and 1.8 to $5.8 \%$ in developing countries (7). 


\section{Direct-Acting Antiviral (DAA) Medications}

General Information. The initial monotherapy trials of the first generation NS3/4A protease inhibitors were hindered by rapid selection of resistant variants (2). It was also recognized that the standard of care with injected interferon-alpha combined with ribavirin showed activity against protease inhibitor-resistant HCV. This antiviral therapy inhibits HCV replication, not by binding viral proteins, but by induction of antiviral and immunomodulatory effects. Therefore, the key first step in the now recognized oral therapeutic revolution against $\mathrm{HCV}$ infection was the addition of a NS3/4a protease inhibitor to the standard treatment of peginterferon and ribavirin. This strategy led to enhanced rates of HCV sustained virological rate (SVR) from approximately $45 \%$ to $75 \%$ among adult patients with genotype $1 \mathrm{HCV}$ infection (2). There was proof, therefore, that use of DAAs was clinically effective but the sideeffects of interferon-based regimens including cytopenias, autoimmune reactions and depression remained a limiting problem. The subsequent rapid development of HCV NS5A inhibitors, and nucleoside/non-nucleoside NS5B polymerase inhibitors since 2011 is well reported, including new treatment combinations of HCV DAAs. The most recent adult clinical trials have proved that interferon-free, all oral DAA combinations are consistently capable of producing SVR rates $>90 \%$ (as defined by HCV RNA clearance 12-weeks after cessation of treatment; SVR12) (810). High optimism now exists for the global eradication of HCV infection (11). Table 1 summarizes DAAs approved to date in adults. First generation NS3/4 protease inhibitors such as telaprevir and boceprevir are no longer used because of their higher rates of adverse effects and treatment failures in real-world settings (11).

Results of Clinical Trials in Adults and 'Real-World' experience. In late 2013, sofosbuvir, a NS5B polymerase inhibitor (in combination with ribavirin), became the first 
Federal Drug Administration (FDA)-approved DAA that allowed interferon-free therapy for HCV genotypes 2 and 3 (12). Then, in early 2014, the 'all oral' DAA combination of sofosbuvir with a NS5A inhibitor, daclatasvir, was used for treatment-naïve adults with HCV genotypes 1, 2, and 3 and $\mathrm{HCV}$ genotype 1 patients and previous protease inhibitor failure; SVR12 rates were excellent at $>93 \%$ (13). In a subsequent trial, previous non-responders infected with HCV genotype 1 treated with sofosbuvir and an NS3/4A protease inhibitor, simeprevir, with or without ribavirin achieved excellent SVR12 rates of $90 \%$ and $94 \%$, respectively; in addition to a good safety profile and no apparent requirement for ribavirin (14). Sofosbuvir and ledipasvir, another NS5A inhibitor, were co-formulated in 2016, as was the regimen of paritaprevir (NS3/4 protease inhibitor boosted with ritonavir), ombitasvir (NS5A inhibitor) and dasabuvir (NS5B inhibitor).

Two phase-3 studies were reported in August 2016. In OPTIMIST 1, sofosbuvir and simeprevir were combined to treat a large group of HCV-genotype 1 adults without cirrhosis who were treatment naïve and treatment experienced (15). This regimen for 12-weeks was highly effective in the treatment of genotype 1a infected individuals without cirrhosis. The overall SVR12 was 97\%, superior to the historic rate of $87 \%$. In OPTIMIST 2 (16), the same 12-week regimen in an open-label fashion was used, but the adult HCV genotype 1a patients had well-compensated cirrhosis. In this difficult-to-treat group, the SVR12 was $83 \%$, and emergent resistance-associated variants (RAVs) occurred based on treatment breakthroughs confirmed to be attributable to NS3/4a region mutation. In a recent phase-3 DAA trial of untreated and treatment-experienced adults with HCV genotypes 1, 2, 4, 5 and 6, the use of fixed-dose combination sofosbuvir and velpatasvir (a novel NS5A inhibitor) led to an overall high rate of SVR12 of $99 \%$ (17). Similarly, adults given this combination regimen with 'difficult to treat' 
Topic of the Month: JPGN 16-562

HCV genotypes 2 and 3, achieved SVR12 of 99\% and 96\%, respectively (18). Thus, these trials showed that treatment with sofosbuvir-velpatasvir were effective regardless of HCV genotype (pan-genotypic). Comprehensive, up-to-date $\mathrm{HCV}$ treatment recommendations sanctioned by the American Association for the Study of Liver Diseases, Infectious Disease Society of America, European and World Health Organization are available (8-10).

The potential selection bias inherent to all registration studies raises questions about generalizing trial derived DAA efficacy and safety results to the adult HCV population at large. Thus, several HCV DAA 'real-world' registries including HCV-TARGET, ANRS CO22 Hepather Cohort and the U.S. Veterans Study were developed. Initial results from these large adult cohorts that include patients with advanced liver disease, and those with contraindications to interferon and/or ribavirin and co-morbidities confirm that DAA therapy is highly effective and safe outside of the registration studies (19-21).

\section{Present Standard Treatment of HCV Infection in Children}

The present standard of care for children and adolescents less than 18 years-old with chronic HCV infection is pegylated interferon with ribavirin (22-25). This combination therapy results in a SVR of 52\% of children with HCV genotype 1 and 4, after 48-weeks of therapy; and, $89 \%$ for children with genotypes 2 and 3, after 24-weeks of treatment. However, interferon regimens are of long duration, require injections to deliver medication, and drug effectiveness is limited by the side-effect profile that includes cytopenias, autoimmunity and depression. And, it is now clear, as reviewed above, that the non-interferon, oral DAA medications are highly effective and well tolerated in adults. There is only very limited data on DAA treatment in children. In the first 'all oral' pediatric trial reported, the use of fixed-dose combination sofosbuvir/ledipasvir in children 12-17 years-old infected with HCV genotype 1 demonstrated 
satisfactory pharmacokinetic and tolerability profiles and high rates of SVR12 (98\%) (26).

Similar excellent results were recently reported in abstract form for the combination of sofosbuvir-ribavirin in adolescents with HCV genotype 2 and 3 infection with SVR of 100\% and 97\%, respectively (27).

\section{Treatment of Children with DAAs: is Delay of Therapy Justified?}

Our limited ability to risk-stratify patients with minimal HCV disease coupled with a poor understanding of the dynamics progression of chronic $\mathrm{HCV}$ infection makes it important to recognize the extraordinary efficacy of new, oral DAA therapy for ALL patients with HCV. In fact, the DAA therapeutic revolution raises the prospect of genuinely eradicating $\mathrm{HCV}$ infection. In order to achieve this goal; however, DAA cost analyses will be essential in developed and resource-limited countries, in addition to creation of effective HCV screening programs with effective linkage to quality patient care facilities.

In terms of cost-effectiveness, patient age is an important determining factor (28). Treatment of younger individuals i.e., children, is more cost-saving per quality-adjusted-life year than treating adults, and pediatric weight-based DAA dosing translates into a need for less medication with subsequent reduced drug costs. Also, the projected long-term cost of medical follow-up is probably less in children than in adults (29). Furthermore, early-in-life eradication of HCV may be important before high-risk adolescent behaviors such as injection-drug use, obtaining tattoos in an unregulated setting and unprotected sexual activity contribute to the spread of infection. The commitment of motivated parents to adhere to a prescribed antiviral regimen, enhances pediatric compliance that is critically important to achieve therapeutic success (30). Moreover, co-morbidities such HIV co-infection, renal impairment, illegal drug and alcohol misuse, and decompensated cirrhosis that may portend unfavourable treatment outcomes are 
uncommon in children (29). Curing HCV infection in females before they achieve childbearing age might be the best approach to preventing future vertical transmission. Thus, HCV screening of high-risk mothers and their infants should be performed.

Have children been left behind in the HCV treatment revolution? As noted, partial results of only two DAA pediatric trials have been reported to date 26,27 ) while others are ongoing. In addition, several developmental plans to support authorization of combination DAA regimens for children have been submitted by pharmaceutical companies to the European Medicines Agency (EMA) and FDA, but the current timelines for completion of approved plans are in the distant future. For example, the estimated date of completion of a sofosbuvir with ribavirin trial for children infected with HCV genotype 2 and 3 is April 2018 (clinicaltrial.gov identifier NCT02175758). For children with HCV genotype 1 and 4 infection, the estimated date of completion of a sofosbuvir and ledipasvir +/- ribavirin trial (identifier NCT02249182) and a paritaprevir boosted with ritonavir/ombitasvir+/- dasabuvir (an NS5B inhibitor) +/-ribavirin trial (identifier NCT02486406) are May 2018 and September 2019, respectively. The long delay is a direct consequence of multiple barriers which impede rapid drug registration and pediatricregulatory requirements. In many high-HCV prevalent nations, trial recruitment is also hindered by regulations that require either drug approval by EMA and/or FDA. To hasten pediatric access to new DAA therapies, in December 2014 the EMA approved a strategic approach for the registration of children's DAA regimens (31). Because of the high efficacy and excellent DAA safety profile in adults, new treatment regimens can now be approved for children in Europe on the basis of phase 1 and 2 trial studies of only 30 to 40 participants. Also, in May 2016, the FDA recommended that pharmaceutical companies focus on development of DAAs for children, especially for the best studied regimens based on collaborative adult data (32). The FDA also 
advocated for earlier development of pediatric DAA drugs; and, more importantly, for the inclusion of younger adolescents in adult phase 3 trials (40). The above will hopefully accelerate registration of DAA regimens for all children.

The cure of HCV infection is possible for almost all individuals treated with interferonfree, oral DAA combination regimens. Global elimination of hepatitis $C$ virus is presently hampered by the above noted barriers, especially poor screening and identification of infected people, and the high price tag of expensive, but very efficacious and safe DAA drugs. We believe that children should be an integral component of the public health approach required for success in realizing global eradication of $\mathrm{HCV}$ infection. The close interplay between pediatric researchers, appropriate healthcare regulators and pharmaceutical industry leaders is essential in order to accelerate the opportunity for children to receive curative oral DAA therapy to combat chronic hepatitis $\mathrm{C}$ virus infection. 


\section{References}

1. Choo QL, Kuo G Weiner AJ, et al. Isolation of ac DNA clone derived from blood borne non-A, nonB viral hepatitis genome. Science 1989; 294:359-362.

2. Chung, RT and Baumert, TF. Curing chronic hepatitis C: The arc of medical triumph. N Engl J Med $2014 ; 370: 1576-1578$.

3. Schaefer EAK, Chung RT. Anti-hepatitis C virus drugs in development. Gastroenterology 2012:142:1340-50.

4. Messina JP, Humphreys I, Flaxsman A, et. al. Global distribution and prevalence of hepatitis C virus genotype. Hepatology 2015;61:77-87

5. Rein DB, Wittenborn IS, Weinbaum CM, et al. Dig Liver Dis 2011;43:66-72 Forecasting the morbidity and mortality associated with prevalent cases of pre-cirrhotic chronic hepatitis $\mathrm{C}$ in the United States.

6. Gower F, Estes CC, Hindeman S, et al. Global epidemiology and genotype distribution for the hepatitis C virus infection. J Hepatol 2014;545-57

7. El-Shabrawi, MH and Kamal, NM. Burden of pediatric hepatitis C World J Gastroenterol 2013;19:7880-7888

8. http://www.who.int/hiv/pub/hepatitis/hepatitis-c-guidelines/en/ ; accessed July 2016.

9. American Association for Ne Study of Liver Disease. HCV Guidance: Recommendations for testing, managing and treating hepatitis C. www.hcvguidelines.org/full-report-view. Accessed December, 2016.

10. European Association for the study of Liver. Clinical March Guidelines: Recommendation on treatment of hepatitis C 2015. www.easl.eu/research/our-contributions/clinicalpracticeguidelines/detail/recommendations-on-treatment-of-hepatitis-c-2015. Accessed May 5, 2015.

11. Vargas, HE. Hepatitis C treatment in 2016. Reason to be an optimist. Hepatology 2016;64:330-331.

12. Lewib E, Mangea A, Wyles D, et al. Sofosbuvir for previously untreated chronic hepatitis C infection. N Engl J Med 2013;368:1878-1887 
Topic of the Month: JPGN 16-562

13. Sulkowski MS, Gardner DF, Rodriguez-Torres VM, et al. DacIatasvir plus sofosbuvir for previously treated and untreated chronic HCV infection. N Engl J Med 2014;370:211-221.

14. Lawitz E. Sulkowski MS, Ghalib R, et al. Simeprevir plus sofosbuvir, with or without ribavirin to treat chronic infection with hepatitis $\mathrm{C}$ virus genotype 1 in non-responders to pegylated interferon and ribavirin and treatment naïve patients: The COSMOS randomised trial. Lancet 2014;384:1756-1765.

15. Kwo P, Gitlin N, Nahass R, et al. Simeprevir plus sofosbuvir (12 and 8 weeks) in HCV genotype 1 infected patients without cirrhosis: OPTIMIST-1, a phase 3, randomized study. Hepatology 2016; 64:370-380.

16. Lawitz E, Matusow G, DeJesus E, et al. Simeprevir plus sofosbuvir in patients with chronic hepatitis C virus genotype 1 infection and cirrhosis: A phase 3 study (OPTIMIST-2). Hepatology 2016; 64:360-369.

17. Feld, JJ, Jacobson C, Hezode T, et al. Sofosbuvir and Velpatasvir for HCV genotype 1, 2, 4, 5, and 6 infection. N Engl J Med 2015; 373:2599-2607.

18. Foster GR, Afdhal N, Roberts SK, et al. Sofosbuvir and Velpatasvir for HCV genotype 2 and 3 infection. N Engl J Med 2015:373:2608-2617.

19. Sulkowski MS, Vargas HE, Di Bisceglie AM, et al. Effectiveness of Simeprevir Plus Sofosbuvir, With or Without Ribavirin, in Real-World Patients With HCV Genotype 1 Infection. Gastroenterology 2016;150:419-29.

20. Pol S, Bourliere M, Lucier S, et al. Safety and efficacy of the combination daclatasvir sofosbuvir in HCV genotype 1-mono-infected patients from the French Observational cohort ANRS CO22. J Hepatol 2015;62:S258.

21. Backus LI, Belperio PS, Shahoumian TA, et al. Effectiveness of sofosbuvir-based regimens in genotype 1 and 2 hepatitis C virus infection in 4026 U.S. Veterans. Aliment Pharmacol Ther 2015;42:559-73. 
22. Druyts E, Thorlund K, Wu P, et al. Efficacy and safety of pegylated interferon alfa-2a or alfa-2b plus ribavirin for the treatment of chronic hepatitis $\mathrm{C}$ in children and adolescents: a systematic review and meta-analysis. Clin Infect Dis 2013;56:961-7

23. Schwarz KB, Gonzalez-Peralta RP, Murray KF, et al. The combination of ribavirin and peginterferon is superior to peginterferon and placebo for children and adolescents with chronic hepatitis $\mathrm{C}$. Gastroenterology 2011;140:450-8.e1

24. Wirth S, Ribes-Koninckx C, Calzado MA, et al. High sustained virologic response rates in children with chronic hepatitis C receiving peginterferon alfa-2b plus ribavirin. J Hepatol 2010;52:501-7.

25. Schwarz KB, Molleston JP, Jonas MM, et al. Children treated with pegylated interferon alfa-2a \pm ribavirin for chronic hepatitis C. J Peds Gastroenterol Nutr 2016; 62:93-96.

26. Balistreri WF, Murray KF, Rosenthal P, et. al. Hepatology 2016 (in press).

27. Schwarz K, Rosenthal P, Gonzalez-Peralta RP, et al. Hepatology 2016; 64:349A.

28. Pho, MT and Linas, BP. Valuing cure: fast affections and coverage decrements for hepatitis C therapy. Hepatology 2014;60:12-13.

29. Bortolotti F, Verucci G, Camma C, at al. Long-term course of chronic hepatitis C in children: from viral clearance to end-stage liver disease. Gastroenterology 2008;134:1900-1907.

30. Zoulim F, Liang TJ, Gerbes AL, et al. Hepatitis C virus treatment in the real world: optimizing treatment and access to therapies. Gut 2015;64:1824-33.

31. www.ema.europa.eu/ema/index.jsp?curl=pages/news_and_events/events/2014/12/event_detail_00107 4.jsp\&mid=WC0b01ac058004d5c3; accessed July 2016.

32. http://www.fda.gov/ucm/groups/fdagov-public/@fdagov-drugs-gen/documents/document/ucm 225333.pdf; accessed July 2016. 
Table 1. New direct acting antivirals approved or in the pipeline for treating HCV infection.

\begin{tabular}{|c|c|c|c|}
\hline Class & Suffix & Approved & In the pipeline \\
\hline NS3/4A protease inhibitors & - previr & $\begin{array}{l}\text { Boceprevir (taken off the market) } \\
\text { Telaprevir (taken off the market) } \\
\text { Simeprevir } \\
\text { Asunaprevir } \\
\text { Paritaprevir (boosted with ritonavir) } \\
\text { Grazoprevir }\end{array}$ & $\begin{array}{l}\text { Voxilaprevir } \\
\text { Glecaprevir } \\
\text { Sovaprevir }\end{array}$ \\
\hline NS5A inhibitors & - asvir & $\begin{array}{l}\text { Daclatasvir } \\
\text { Ledipasvir } \\
\text { Ombitasvir } \\
\text { Elbasvir } \\
\text { Velpatasvir }\end{array}$ & $\begin{array}{l}\text { Odalasvir } \\
\text { Pibrentasvir } \\
\text { Ruzasvir } \\
\text { Samatasvir }\end{array}$ \\
\hline NS5B polymerase inhibitors & - buvir & $\begin{array}{l}\text { Sofosbuvir } \\
\text { Dasabuvir }\end{array}$ & $\begin{array}{l}\text { Beclabuvir } \\
\text { MK-3682 } \\
\text { ACH-3422 } \\
\text { AL-335 } \\
\text { GS-9669 } \\
\text { MK-8876 }\end{array}$ \\
\hline
\end{tabular}

